\title{
Soft $\mathrm{g}^{\star}$-Closed Sets in Soft Minimal Spaces
}

\author{
*R. Gowri and S. Vembu \\ Department of Mathematics, Government College for Women (A), Kumbakonam, India \\ Email: *gowrigck@rediffmail.com, vembuchandrasekaran@gmail.com
}

\begin{abstract}
In this paper, we define soft $g^{*}$-closed and soft $g^{*}$-open sets in soft minimal spaces which are defined over an initial universe with a fixed set of parameters. Further, we discuss basic properties of their sets.
\end{abstract}

Keywords: soft sets, soft minimal space, $s g^{*} \widetilde{m}$-closed sets, $s g^{*} \widetilde{m}$-open sets.

AMS Subject Classification: 54A10, 54C08

\section{Introduction:}

The concept of soft set theory as a new mathematical tool was initiated by Molodtsov [9]. M. Shabir and M. Naz [13] introduced soft topological spaces and the notions of soft closed sets, soft open sets, soft closure, soft interior and soft neighborhood of a point. Levine [8] initiated the study of generalized closed and open sets in topological spaces. Noiri [11] introduced the notion of generalized m-closed (briefly gm-closed) sets and tried to unify certain types of modifications of g-closed sets. Boonpok [1] introduced biminimal structure spaces and studied $\mathrm{m}^{1} \mathrm{~m}^{2}{ }_{\mathrm{X}}$-closed and $\mathrm{m}_{\mathrm{X}}^{1} \mathrm{~m}^{2} \mathrm{x}^{-}$open sets in biminimal structure spaces. Recently, in 2015, Gowri and Vembu [4] introduced and studied soft minimal and soft biminimal spaces.

In this present study, we discuss soft $\mathrm{g}^{\star}$-closed and soft $\mathrm{g}^{\star}$-open sets in soft minimal spaces and obtain the basic results and properties.

\section{Preliminaries:}

Definition 2.1 [9]: Let $U$ be an initial universe and $E$ is a set of parameters. Let $P(U)$ denotes the power set of $U$ and $A$ is a nonempty subset of E. A pair (F, A) is called a soft set over $U$, where $F$ is a mapping given by $F: A \rightarrow P(U)$.

In other words, a soft set over $U$ is a parameterized family of subsets of the universe $U$. For $\varepsilon \in A$. F $(\varepsilon)$ may be considered as the set of $\varepsilon$ - approximate elements of the soft set $(\mathrm{F}, \mathrm{A})$.

Definition 2.2 [4]: Let $\mathrm{X}$ be an initial universe set, $\mathrm{E}$ be the set of parameters and $\mathrm{A}$ be the soft subset of $\mathrm{E}\left(\mathrm{A} \widetilde{\subseteq}\right.$ ). Let $\mathrm{F}_{\mathrm{A}}$ be a nonempty soft set over $\mathrm{X}$ and $\tilde{p}\left(\mathrm{~F}_{\mathrm{A}}\right)$ is the soft 
power set of $\mathrm{F}_{\mathrm{A}}$. A subfamily $\widetilde{m}$ of $\tilde{p}\left(\mathrm{~F}_{\mathrm{A}}\right)$ is called a soft minimal over $\mathrm{X}$ if $\mathrm{F}_{\Phi} \in \widetilde{m}$ and $\mathrm{F}_{\mathrm{A}} \in \widetilde{m}$.

$\left(\mathrm{F}_{\mathrm{A}}, \widetilde{m}\right)$ or $(\mathrm{X}, \widetilde{m}, \mathrm{E})$ is called a soft minimal space over $\mathrm{X}$. Each member of $\widetilde{m}$ is said to be $\widetilde{m}$ - soft open set and the complement of a $\widetilde{m}$ - soft open set is said to be $\widetilde{m}$ soft closed set over X.

\section{Example 2.3:}

Let $\mathrm{U}=\left\{\mathrm{u}_{1}, \mathrm{u}_{2}\right\}, \mathrm{E}=\left\{\mathrm{x}_{1}, \mathrm{x}_{2}, \mathrm{x}_{3}\right\}, \mathrm{A}=\left\{\mathrm{x}_{1}, \mathrm{x}_{2}\right\} \widetilde{\subseteq} \mathrm{E}$ and $\mathrm{F}_{\mathrm{A}}=\left\{\left(\mathrm{x}_{1}\left\{\mathrm{u}_{1}, \mathrm{u}_{2}\right\}\right),\left(\mathrm{x}_{2}\left\{\mathrm{u}_{1}, \mathrm{u}_{2}\right\}\right)\right\}$. Then

$\mathrm{F}_{\mathrm{A} 1}=\left\{\left(\mathrm{x}_{1}\left\{\mathrm{u}_{1}\right\}\right)\right\}, \mathrm{F}_{\mathrm{A} 2}=\left\{\left(\mathrm{x}_{1}\left\{\mathrm{u}_{2}\right\}\right)\right\}, \mathrm{F}_{\mathrm{A} 3}=\left\{\left(\mathrm{x}_{1}\left\{\mathrm{u}_{1}, \mathrm{u}_{2}\right\}\right)\right\}$,

$\mathrm{F}_{\mathrm{A} 4}=\left\{\left(\mathrm{x}_{2}\left\{\mathrm{u}_{1}\right\}\right)\right\}, \mathrm{F}_{\mathrm{A} 5}=\left\{\left(\mathrm{x}_{2}\left\{\mathrm{u}_{2}\right\}\right)\right\}, \mathrm{F}_{\mathrm{A} 6}=\left\{\left(\mathrm{x}_{2}\left\{\mathrm{u}_{1}, \mathrm{u}_{2}\right\}\right)\right\}$,

$\mathrm{F}_{\mathrm{A} 7}=\left\{\left(\mathrm{x}_{1}\left\{\mathrm{u}_{1}\right\}\right),\left(\mathrm{x}_{2}\left\{\mathrm{u}_{1}\right\}\right)\right\}, \mathrm{F}_{\mathrm{A} 8}=\left\{\left(\mathrm{x}_{1}\left\{\mathrm{u}_{1}\right\}\right),\left(\mathrm{x}_{2}\left\{\mathrm{u}_{2}\right\}\right)\right\}$

$\mathrm{F}_{\mathrm{A} 9}=\left\{\left(\mathrm{x}_{1}\left\{\mathrm{u}_{1}\right\}\right),\left(\mathrm{x}_{2}\left\{\mathrm{u}_{1}, \mathrm{u}_{2}\right\}\right)\right\}, \mathrm{F}_{\mathrm{A} 10}=\left\{\left(\mathrm{x}_{1}\left\{\mathrm{u}_{2}\right\}\right),\left(\mathrm{x}_{2}\left\{\mathrm{u}_{1}\right\}\right)\right\}$

$\mathrm{F}_{\mathrm{A} 11}=\left\{\left(\mathrm{x}_{1}\left\{\mathrm{u}_{2}\right\}\right),\left(\mathrm{x}_{2}\left\{\mathrm{u}_{2}\right\}\right)\right\}, \mathrm{F}_{\mathrm{A} 12}=\left\{\left(\mathrm{x}_{1}\left\{\mathrm{u}_{2}\right\}\right),\left(\mathrm{x}_{2}\left\{\mathrm{u}_{1}, \mathrm{u}_{2}\right\}\right)\right\}$

$\mathrm{F}_{\mathrm{A} 13}=\left\{\left(\mathrm{x}_{1}\left\{\mathrm{u}_{1}, \mathrm{u}_{2}\right\}\right),\left(\mathrm{x}_{2}\left\{\mathrm{u}_{1}\right\}\right)\right\}, \mathrm{F}_{\mathrm{A} 14}=\left\{\left(\mathrm{x}_{1}\left\{\mathrm{u}_{1}, \mathrm{u} 2\right\}\right),\left(\mathrm{x}_{2}\left\{\mathrm{u}_{2}\right\}\right)\right\}$

$\mathrm{F}_{\mathrm{A} 15}=\mathrm{F}_{\mathrm{A},} \mathrm{F}_{\mathrm{A} 16}=\mathrm{F}_{\Phi}$ are all soft subset of $\mathrm{F}_{\mathrm{A}}$,

$$
\text { Soft minimal }(\widetilde{m})=\left\{\mathrm{F} \Phi, \mathrm{F}_{\mathrm{A} 1}, \mathrm{~F}_{\mathrm{A} 3}, \mathrm{~F}_{\mathrm{A} 7}, \mathrm{~F}_{\mathrm{A} 11}, \mathrm{~F}_{\mathrm{A} 13}, \mathrm{~F}_{\mathrm{A}}\right\} \text {. }
$$

Definition 2.4 [4]: Let $\left(\mathrm{F}_{\mathrm{A}}, \widetilde{m}\right)$ be a soft minimal space with nonempty soft set $\mathrm{F}_{\mathrm{A}}$ is said to have property $B$ if the union of any family of soft subsets belonging to $\widetilde{m}$ belongs to $\widetilde{m}$

Definition 2.5 [4]: Let $\left(\mathrm{F}_{\mathrm{A}}, \widetilde{m}\right)$ be a soft minimal space over $\mathrm{X}$. For a soft subset $\mathrm{F}_{B}$ of $\mathrm{F}_{\mathrm{A}}$ the $\widetilde{m}$-soft closure of $\mathrm{F}_{\mathrm{B}}$ and $\widetilde{m}$-soft interior of $\mathrm{F}_{\mathrm{B}}$ are defined as follows:

(1) $\widetilde{m} \mathrm{Cl}\left(\mathrm{F}_{\mathrm{B}}\right)=\bigcap\left\{\mathrm{F}_{\alpha}: \mathrm{F}_{\mathrm{B}} \widetilde{\subseteq} \mathrm{F}_{\alpha}, \mathrm{F}_{\mathrm{A}^{-}}-\mathrm{F}_{\alpha} \in \widetilde{m}\right\}$,

(2) $\tilde{m}$ Int $\left(\mathrm{F}_{\mathrm{B}}\right)=\cup\left\{\mathrm{F}_{\beta}: \mathrm{F}_{\beta} \widetilde{\subseteq} \mathrm{F}_{\mathrm{B}}, \mathrm{F}_{\beta} \in \tilde{m}\right\}$.

\section{Lemma 2.6 [4]:}

Let $\left(\mathrm{F}_{\mathrm{A}}, \widetilde{m}\right)$ be a soft minimal space over $\mathrm{X}$. For a soft subset $\mathrm{F}_{\mathrm{B}}$ and $\mathrm{F}_{\mathrm{C}}$ of $\mathrm{F}_{\mathrm{A}}$, the following properties hold:

(1) $\tilde{m} \mathrm{Cl}\left(\mathrm{F}_{\mathrm{A}}-\mathrm{F}_{\mathrm{B}}\right)=\mathrm{F}_{\mathrm{A}}-\tilde{m} \operatorname{Int}\left(\mathrm{F}_{\mathrm{B}}\right)$ and $\widetilde{m} \operatorname{Int}\left(\mathrm{F}_{\mathrm{A}}-\mathrm{F}_{\mathrm{B}}\right)=\mathrm{F}_{\mathrm{A}}-\tilde{m} \mathrm{Cl}\left(\mathrm{F}_{\mathrm{B}}\right)$,

(2) If $\left(\mathrm{F}_{\mathrm{A}}-\mathrm{F}_{\mathrm{B}}\right) \in \widetilde{m}$, then $\widetilde{m} \mathrm{Cl}\left(\mathrm{F}_{\mathrm{B}}\right)=\mathrm{F}_{\mathrm{B}}$ and if $\mathrm{F}_{\mathrm{B}} \in \widetilde{m}$, then $\widetilde{m}$ Int $\left(\mathrm{F}_{\mathrm{B}}\right)=\mathrm{F}_{\mathrm{B}}$,

(3) $\widetilde{m} \mathrm{Cl}(\mathrm{F} \Phi)=\mathrm{F} \Phi, \tilde{m} \mathrm{Cl}\left(\mathrm{F}_{\mathrm{A}}\right)=\mathrm{F}_{\mathrm{A}}, \widetilde{m} \mathrm{Int}(\mathrm{F} \Phi)=\mathrm{F} \Phi$ and $\widetilde{m} \operatorname{Int}\left(\mathrm{F}_{\mathrm{A}}\right)=\mathrm{F}_{\mathrm{A}}$,

(4) If $\mathrm{F}_{\mathrm{B}} \widetilde{\subseteq} \mathrm{F}_{\mathrm{C}}$ then $\widetilde{m} \mathrm{Cl}\left(\mathrm{F}_{\mathrm{B}}\right) \widetilde{\subseteq} \widetilde{m} \mathrm{Cl}\left(\mathrm{F}_{\mathrm{C}}\right)$ and $\widetilde{m} \mathrm{Int}\left(\mathrm{F}_{\mathrm{B}}\right) \widetilde{\subseteq} \widetilde{m} \mathrm{Cl}\left(\mathrm{F}_{\mathrm{C}}\right)$,

(5) $\mathrm{F}_{\mathrm{B}} \widetilde{\subseteq} \widetilde{m} \mathrm{Cl}\left(\mathrm{F}_{\mathrm{B}}\right)$ and $\widetilde{m} \operatorname{Int}\left(\mathrm{F}_{\mathrm{B}}\right) \widetilde{\subseteq} \mathrm{F}_{\mathrm{B}}$,

(6) $\widetilde{m} \mathrm{Cl}\left(\widetilde{m} \mathrm{Cl}\left(\mathrm{F}_{\mathrm{B}}\right)\right)=\widetilde{m} \mathrm{Cl}\left(\mathrm{F}_{\mathrm{B}}\right)$ and $\widetilde{m} \operatorname{Int}\left(\widetilde{m} \operatorname{Int}\left(\mathrm{F}_{\mathrm{B}}\right)\right)=\widetilde{m} \operatorname{Int}\left(\mathrm{F}_{\mathrm{B}}\right)$. 
Lemma 2.7 [4]: Let $\mathrm{F}_{\mathrm{A}}$ be a nonempty soft set with a soft minimal $\tilde{\text { mon }} \mathrm{F}_{\mathrm{A}}$ satisfying property $B$. For a soft subset $F_{B}$ of $F_{A}$ the following properties hold:

(1) $\mathrm{F}_{\mathrm{B}} \in \widetilde{m}$ if and only if $\widetilde{m}$ Int $\left(\mathrm{F}_{\mathrm{B}}\right)=\mathrm{F}_{\mathrm{B}}$

(2) If $\mathrm{F}_{\mathrm{B}}$ is soft $\tilde{m}$-closed if and only if $\tilde{m} \mathrm{Cl}\left(\mathrm{F}_{\mathrm{B}}\right)=\mathrm{F}_{\mathrm{B}}$

(3) $\tilde{m}$ Int $\left(\mathrm{F}_{\mathrm{B}}\right) \in \widetilde{m}$ and $\tilde{m} \mathrm{Cl}\left(\mathrm{F}_{\mathrm{B}}\right)$ is soft $\tilde{m}$-closed.

Definition 2.8 [5]: A soft subset $\mathrm{F}_{\mathrm{B}}$ of a soft minimal space $\left(\mathrm{F}_{\mathrm{A}}, \tilde{m}\right)$ is said to be soft

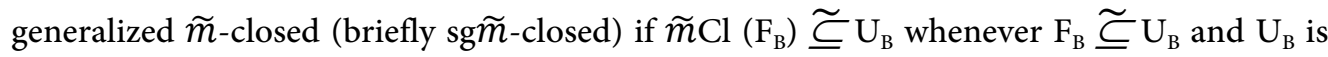
soft $\tilde{m}$-open. The complement of soft generalized $\tilde{m}$-closed set is said to be soft generalized $\tilde{m}$ open (briefly sg $\tilde{m}$-open)

\section{Soft $\mathbf{g}^{\star}$-closed sets in soft minimal space}

Definition 3.1: A soft subset $\mathrm{F}_{\mathrm{B}}$ of a soft minimal space $\left(\mathrm{F}_{\mathrm{A}}, \widetilde{m}\right)$ is said to be soft $\mathrm{g}^{\star} \tilde{m}$ closed set (briefly sg* $\widetilde{m}$-closed set) if $\widetilde{m} \mathrm{Cl}\left(\mathrm{F}_{\mathrm{B}}\right) \widetilde{\subseteq} \mathrm{U}_{\mathrm{B}}$ whenever $\mathrm{F}_{\mathrm{B}} \widetilde{\subseteq} \mathrm{U}_{\mathrm{B}}$ and $\mathrm{U}_{\mathrm{B}}$ is sg $\tilde{m}$ open.

Example 3.2: Let us consider the soft subsets of $F_{A}$ that are given in Example 2.3. Let $\left(\mathrm{F}_{\mathrm{A}}, \widetilde{m}\right)$ be a soft minimal space where $\mathrm{U}=\left\{\mathrm{u}_{1}, \mathrm{u}_{2}\right\}, \mathrm{E}=\left\{\mathrm{x}_{1}, \mathrm{x}_{2}, \mathrm{x}_{3}\right\}, \mathrm{A}=\left\{\mathrm{x}_{1}, \mathrm{x}_{2}\right\} \widetilde{E}$ and $\mathrm{F}_{\mathrm{A}}=\left\{\left(\mathrm{x}_{1}\left\{\mathrm{u}_{1}, \mathrm{u}_{2}\right\}\right),\left(\mathrm{x}_{2}\left\{\mathrm{u}_{1}, \mathrm{u}_{2}\right\}\right)\right\}$. Then $\tilde{m}=\left\{\mathrm{F} \Phi, \mathrm{F}_{\mathrm{A} 6}, \mathrm{~F}_{\mathrm{A} 8}, \mathrm{~F}_{\mathrm{A} 11}, \mathrm{~F}_{\mathrm{A}}\right\}, \widetilde{m}^{\mathrm{C}}=\left\{\mathrm{F} \Phi, \mathrm{F}_{\mathrm{A} 3}, \mathrm{~F}_{\mathrm{A} 10}, \mathrm{~F}_{\mathrm{A} 7}\right.$, $\left.\mathrm{F}_{\mathrm{A}}\right\}$,

sg $\tilde{m}$ - closed sets are $\mathrm{F} \Phi, \mathrm{F}_{\mathrm{A} 1}, \mathrm{~F}_{\mathrm{A} 2}, \mathrm{~F}_{\mathrm{A} 3}, \mathrm{~F}_{\mathrm{A} 4}, \mathrm{~F}_{\mathrm{A} 7}, \mathrm{~F}_{\mathrm{A} 9}, \mathrm{~F}_{\mathrm{A} 10}, \mathrm{~F}_{\mathrm{A} 12}, \mathrm{~F}_{\mathrm{A} 13}, \mathrm{~F}_{\mathrm{A} 14}, \mathrm{~F}_{\mathrm{A}}$ and

$\operatorname{sg}^{\star} \tilde{m}$-closed sets are $\mathrm{F} \Phi, \mathrm{F}_{\mathrm{A} 1}, \mathrm{~F}_{\mathrm{A} 2}, \mathrm{~F}_{\mathrm{A} 3}, \mathrm{~F}_{\mathrm{A} 4}, \mathrm{~F}_{\mathrm{A} 7}, \mathrm{~F}_{\mathrm{A} 10}, \mathrm{~F}_{\mathrm{A} 13}, \mathrm{~F}_{\mathrm{A}}$.

Theorem 3.3: Every soft $\widetilde{m}$-closed set is $\operatorname{sg}^{\star} \widetilde{m}$-closed set.

Proof: Let $\mathrm{F}_{\mathrm{B}}$ be soft $\widetilde{m}$-closed set in $\left(\mathrm{F}_{\mathrm{A}}, \widetilde{m}\right)$ and $\mathrm{U}_{\mathrm{B}}$ is sg $\widetilde{m}$-open such that $\mathrm{F}_{\mathrm{B}} \widetilde{\complement} \mathrm{U}_{\mathrm{B}}$. Then $\tilde{m} \mathrm{Cl}\left(\mathrm{F}_{\mathrm{B}}\right)=\mathrm{F}_{\mathrm{B}}$ and so $\tilde{m} \mathrm{Cl}\left(\mathrm{F}_{\mathrm{B}}\right) \widetilde{\leftarrow} \mathrm{U}_{\mathrm{B}}$. Hence $\mathrm{F}_{\mathrm{B}}$ be sg ${ }^{\star} \widetilde{m}$-closed set.

Theorem 3.4: Every sg* $\widetilde{m}$-closed set is sg $\widetilde{m}$-closed set.

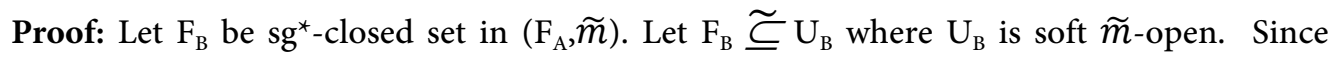
every soft $\tilde{m}$-open set is sg $\tilde{m}$-open set. Since $\mathrm{F}_{\mathrm{B}}$ is sg $^{\star} \tilde{m}$-closed set then we have $\tilde{m}$-Cl $\left(\mathrm{F}_{\mathrm{B}}\right) \widetilde{\simeq} \mathrm{U}_{\mathrm{B}}$. Hence $\mathrm{F}_{\mathrm{B}}$ is sg$\tilde{m}$-closed.

Remark 3.5: From the above observation we get the following implication Soft $\tilde{m}$-closed set $\rightarrow$ sg $^{\star} \tilde{m}$-closed set $\rightarrow$ sg $\tilde{m}$-closed set 
The reverse implications are not true from the following example

Example 3.6: Let us consider the soft subsets of $\mathrm{F}_{\mathrm{A}}$ that are given in Example 2.3. Let $\left(\mathrm{F}_{\mathrm{A}}, \widetilde{m}\right)$ be a soft minimal space where $\mathrm{U}=\left\{\mathrm{u}_{1}, \mathrm{u}_{2}\right\}, \mathrm{E}=\left\{\mathrm{x}_{1}, \mathrm{x}_{2}, \mathrm{x}_{3}\right\}, \mathrm{A}=\left\{\mathrm{x}_{1}, \mathrm{x}_{2}\right\} \widetilde{\complement} \mathrm{E}$ and $\mathrm{F}_{\mathrm{A}}=\left\{\left(\mathrm{x}_{1}\left\{\mathrm{u}_{1}, \mathrm{u}_{2}\right\}\right),\left(\mathrm{x}_{2}\left\{\mathrm{u}_{1}, \mathrm{u}_{2}\right\}\right)\right\}$. Then $\tilde{m}=\left\{\mathrm{F} \Phi, \mathrm{F}_{\mathrm{A} 6}, \mathrm{~F}_{\mathrm{A} 8}, \mathrm{~F}_{\mathrm{A} 11}, \mathrm{~F}_{\mathrm{A}}\right\}$. See Example 3.2. $\mathrm{F}_{\mathrm{A} 2}$ is $\operatorname{sg}^{\star} \tilde{m}$-closed but not soft $\tilde{m}$-closed and $\mathrm{F}_{\mathrm{A} 12}$ is sg $\tilde{m}$-closed but not sg ${ }^{\star} \tilde{m}$-closed.

Theorem 3.7: If $F_{B}$ and $G_{B}$ are $\operatorname{sg}^{\star} \tilde{m}$-closed sets in $\left(\mathrm{F}_{A}, \tilde{m}\right)$ then their union $F_{B} \cup G_{B}$ is also sg* $\widetilde{m}$-closed sets in $\left(\mathrm{F}_{\mathrm{A}}, \widetilde{m}\right)$

Proof: Suppose $\mathrm{F}_{\mathrm{B}}$ and $\mathrm{G}_{\mathrm{B}}$ are $\operatorname{sg}^{\star} \tilde{m}$-closed sets in $\left(\mathrm{F}_{\mathrm{A}}, \widetilde{m}\right)$. Let $\mathrm{U}_{\mathrm{B}} \operatorname{sg} \tilde{m}$-open of $\left(\mathrm{F}_{\mathrm{A}}, \tilde{m}\right)$ such that $\mathrm{F}_{\mathrm{B}} \cup \mathrm{G}_{\mathrm{B}} \simeq \mathrm{U}_{\mathrm{B}}$. Since $\mathrm{F}_{\mathrm{B}} \cup \mathrm{G}_{\mathrm{B}} \widetilde{\subseteq} \mathrm{U}_{\mathrm{B}}$, we have $\mathrm{F}_{\mathrm{B}} \widetilde{\subseteq} \mathrm{U}_{\mathrm{B}}$ and $\mathrm{G}_{\mathrm{B}} \widetilde{\subseteq} \mathrm{U}_{\mathrm{B}}$. Since $\mathrm{F}_{\mathrm{B}}$ and $\mathrm{G}_{\mathrm{B}}$ are sg${ }^{\star} \widetilde{m}$-closed, we have $\tilde{m} \mathrm{Cl}\left(\mathrm{F}_{\mathrm{B}}\right) \simeq \mathrm{U}_{\mathrm{B}}$ and $\widetilde{m} \mathrm{Cl}\left(\mathrm{G}_{\mathrm{B}}\right) \widetilde{\subseteq} \mathrm{U}_{\mathrm{B}}$. Therefore $\tilde{m} \mathrm{Cl}\left(\mathrm{F}_{\mathrm{B}} \cup \mathrm{G}_{\mathrm{B}}\right) \widetilde{\complement} \widetilde{m} \mathrm{Cl}\left(\mathrm{F}_{\mathrm{B}}\right) \cup \widetilde{m} \mathrm{Cl}\left(\mathrm{G}_{\mathrm{B}}\right) \widetilde{\complement} \mathrm{U}_{\mathrm{B}}$. Hence $\mathrm{F}_{\mathrm{B}} \cup \mathrm{G}_{\mathrm{B}}$ is also sg ${ }^{\star} \widetilde{m}$-closed set.

Remark 3.8: The intersection of two $\mathrm{sg}^{\star} \widetilde{m}$-closed sets are need not be $\mathrm{sg}^{\star} \widetilde{m}$-closed as seen from the following example.

Example 3.9: Let us consider the soft subsets of $F_{A}$ that are given in Example 2.3. Let $\left(\mathrm{F}_{\mathrm{A}}, \widetilde{m}\right)$ be a soft minimal space where $\mathrm{U}=\left\{\mathrm{u}_{1}, \mathrm{u}_{2}\right\}, \mathrm{E}=\left\{\mathrm{x}_{1}, \mathrm{x}_{2}, \mathrm{x}_{3}\right\}, \mathrm{A}=\left\{\mathrm{x}_{1}, \mathrm{x}_{2}\right\} \widetilde{\complement} \mathrm{E}$ and $\mathrm{F}_{\mathrm{A}}=\left\{\left(\mathrm{x}_{1}\left\{\mathrm{u}_{1}, \mathrm{u}_{2}\right\}\right),\left(\mathrm{x}_{2}\left\{\mathrm{u}_{1}, \mathrm{u}_{2}\right\}\right)\right\}$. Then $\widetilde{m}=\left\{\mathrm{F} \Phi, \mathrm{F}_{\mathrm{A} 1}, \mathrm{~F}_{\mathrm{A} 2}, \mathrm{~F}_{\mathrm{A} 3}, \mathrm{~F}_{\mathrm{A} 7}, \mathrm{~F}_{\mathrm{A} 6}, \mathrm{~F}_{\mathrm{A} 12}, \mathrm{~F}_{\mathrm{A}}\right\}$. Then $\mathrm{F}_{\mathrm{B}}=\mathrm{F}_{\mathrm{A} 6}$ and $\mathrm{G}_{\mathrm{B}}=\mathrm{F}_{\mathrm{A} 8}$ are sg ${ }^{\star} \tilde{m}$-closed but $\mathrm{F}_{\mathrm{B}} \cap \mathrm{G}_{\mathrm{B}}=\mathrm{F}_{\mathrm{A} 5}$ is not sg ${ }^{\star} \tilde{m}$-closed set.

Theorem 3.10: For each element $(\mathrm{x}, \mathrm{u}) \in\left(\mathrm{F}_{\mathrm{A}}, \widetilde{m}\right)$ then singleton $\{(\mathrm{x}, \mathrm{u})\}$ is sgm-closed or $\{(\mathrm{x}, \mathrm{u})\}^{\mathrm{C}}$ is $\mathrm{sg}^{\star} \widetilde{m}$-closed set.

Proof: Let $(\mathrm{x}, \mathrm{u}) \in\left(\mathrm{F}_{\mathrm{A}}, \widetilde{m}\right)$ and the singleton $\{(\mathrm{x}, \mathrm{u})\}$ is not $\operatorname{sg} \tilde{m}$-closed. Then $\{(\mathrm{x}, \mathrm{u})\}^{\mathrm{C}}$ is not sg $\widetilde{m}$-open set and $\mathrm{F}_{\mathrm{A}}$ is the only sg $\widetilde{m}$-open set which contains $\{(\mathrm{x}, \mathrm{u})\}^{\mathrm{C}}$ and $\{(\mathrm{x}, \mathrm{u})\}^{\mathrm{C}}$ is sg $^{\star} \tilde{m}$-closed set.

Theorem 3.11: Let $\left(\mathrm{F}_{\mathrm{A}}, \tilde{m}\right)$ be soft minimal space. If $\mathrm{F}_{\mathrm{B}}$ is $\mathrm{sg}^{\star} \tilde{m}$-closed, then $\tilde{m} \mathrm{Cl}\left(\mathrm{F}_{\mathrm{B}}\right)-\mathrm{F}_{\mathrm{B}}$ contains no nonempty sg $\tilde{m}$-closed.

Proof: Let $\mathrm{F}_{\mathrm{B}}$ is $\operatorname{sg}^{\star} \tilde{m}$-closed set. Let $\mathrm{H}_{\mathrm{B}}$ is a sg $\tilde{m}$-closed subset of $\tilde{m} \mathrm{Cl}\left(\mathrm{F}_{\mathrm{B}}\right)-\mathrm{F}_{\mathrm{B}}$. That is $\mathrm{H}_{\mathrm{B}} \simeq \widetilde{m} \mathrm{Cl}\left(\mathrm{F}_{\mathrm{B}}\right)-\mathrm{F}_{\mathrm{B}}$. Then $\mathrm{F}_{\mathrm{B}} \widetilde{\subseteq} \mathrm{H}_{\mathrm{B}}{ }^{\mathrm{C}}, \mathrm{H}_{\mathrm{B}}{ }^{\mathrm{C}}$ is sg$\tilde{m}$ open and hence, $\widetilde{m} \mathrm{Cl}\left(\mathrm{F}_{\mathrm{B}}\right) \widetilde{\subseteq} \mathrm{H}_{\mathrm{B}}{ }^{\mathrm{C}}$.

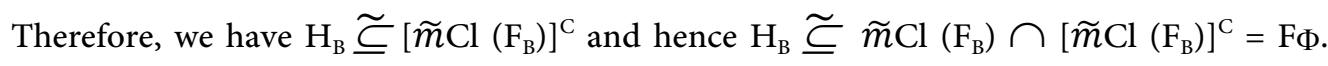
Hence $\mathrm{H}_{\mathrm{B}}=\mathrm{F} \Phi$. Therefore $\tilde{m} \mathrm{Cl}\left(\mathrm{F}_{\mathrm{B}}\right)-\mathrm{F}_{\mathrm{B}}$ contains no nonempty sg $\tilde{m}$-closed. 
Corollary 3.12: A soft minimal $\widetilde{m}$ on a nonempty soft set $\mathrm{F}_{\mathrm{A}}$ satisfying property $\mathrm{B}$. If $\mathrm{F}_{\mathrm{B}}$ is sg* $\widetilde{m}$-closed in $\left(\mathrm{F}_{\mathrm{A}}, \widetilde{m}\right)$, then $\mathrm{F}_{\mathrm{B}}$ is soft $\widetilde{m}$ - closed if and only if $\widetilde{m} \mathrm{Cl}\left(\mathrm{F}_{\mathrm{B}}\right)-\mathrm{F}_{\mathrm{B}}$ is sg $\tilde{m}$-closed.

Proof: If $\mathrm{F}_{\mathrm{B}}$ is soft $\widetilde{m}$-closed, then $\widetilde{m} \mathrm{Cl}\left(\mathrm{F}_{\mathrm{B}}\right)=\mathrm{F}_{\mathrm{B}}$. That is $\widetilde{m} \mathrm{Cl}\left(\mathrm{F}_{\mathrm{B}}\right)-\mathrm{F}_{\mathrm{B}}=\mathrm{F} \Phi$ and hence $\tilde{m} \mathrm{Cl}\left(\mathrm{F}_{\mathrm{B}}\right)-\mathrm{F}_{\mathrm{B}}$ is sg $\tilde{m}$-closed.

Conversely, if $\tilde{m} \mathrm{Cl}\left(\mathrm{F}_{\mathrm{B}}\right)-\mathrm{F}_{\mathrm{B}}$ is sg $\tilde{m}$-closed, then by Theorem 3.11, $\tilde{m} \mathrm{Cl}\left(\mathrm{F}_{\mathrm{B}}\right)-\mathrm{F}_{\mathrm{B}}=\mathrm{F} \Phi$ Since $\mathrm{F}_{\mathrm{B}}$ is sg ${ }^{\star} \widetilde{m}$-closed. Therefore, $\mathrm{F}_{\mathrm{B}}$ is soft $\widetilde{m}$ - closed.

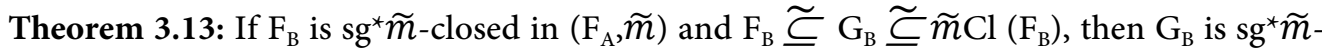
closed.

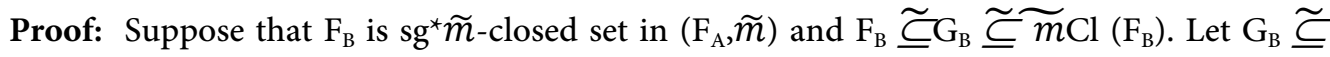
$\mathrm{U}_{\mathrm{B}}$ and $\mathrm{U}_{\mathrm{B}}$ is sg $\widetilde{m}$-open in $\left(\mathrm{F}_{\mathrm{A}}, \widetilde{m}\right)$. Since $\mathrm{F}_{\mathrm{B}} \widetilde{\complement} \mathrm{G}_{\mathrm{B}}$ and $\mathrm{G}_{\mathrm{B}} \widetilde{\subseteq} \mathrm{U}_{\mathrm{B}}$, we have $\mathrm{F}_{\mathrm{B}} \widetilde{\subseteq} \mathrm{U}_{\mathrm{B}}$. Since $\mathrm{F}_{\mathrm{B}}$

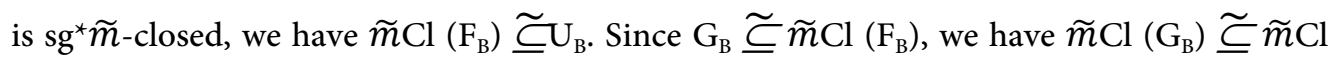
$\left(\mathrm{F}_{\mathrm{B}}\right) \widetilde{\subseteq} \mathrm{U}_{\mathrm{B}}$. Therefore $\mathrm{G}_{\mathrm{B}}$ is sg ${ }^{\star} \widetilde{m}$-closed set.

Definition 3.14: A soft subset $F_{B}$ of a soft minimal space $\left(F_{A}, \tilde{m}\right.$ is said to be soft $g^{\star} \tilde{m}$-open (briefly $\operatorname{sg}^{\star} \widetilde{m}$-open) if its complement is $\operatorname{sg}^{\star} \widetilde{m}$-closed.

Theorem 3.15: A soft subset $\mathrm{F}_{\mathrm{B}}$ of a soft minimal space $\left(\mathrm{F}_{\mathrm{A}}, \tilde{m}\right)$ is $\mathrm{sg}^{\star} \tilde{m}$-open if and only if $\mathrm{H}_{\mathrm{B}} \widetilde{\complement} \tilde{m}$ Int $\left(\mathrm{F}_{\mathrm{B}}\right)$ whenever $\mathrm{H}_{\mathrm{B}} \widetilde{\complement} \mathrm{F}_{\mathrm{B}}$ and $\mathrm{H}_{\mathrm{B}}$ is sg $\tilde{m}$-closed.

Proof: Suppose that $\mathrm{F}_{\mathrm{B}}$ is $\operatorname{sg}^{\star} \tilde{m}$-open. Let $\mathrm{H}_{\mathrm{B}}$ be a sg $\tilde{m}$-closed set such that $\mathrm{H}_{\mathrm{B}} \widetilde{\complement} \mathrm{F}_{\mathrm{B}}$. Then $\mathrm{F}_{\mathrm{B}}{ }^{\mathrm{C}} \widetilde{\subseteq} \mathrm{H}_{\mathrm{B}}{ }^{\mathrm{C}}$ and $\mathrm{H}_{\mathrm{B}}{ }^{\mathrm{C}}$ is sg $\widetilde{m}$-open, we have $\mathrm{F}_{\mathrm{B}}{ }^{\mathrm{C}}$ is sg${ }^{\star} \widetilde{m}$-closed. Hence, $\widetilde{m} \mathrm{Cl}\left(\mathrm{F}_{\mathrm{B}}\right)^{\mathrm{C}} \widetilde{\subseteq} \mathrm{H}_{\mathrm{B}}{ }^{\mathrm{C}}$.

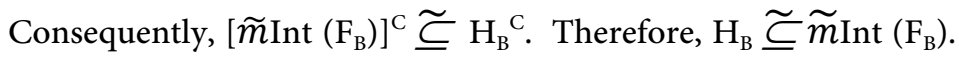

Conversely, assume that $\mathrm{H}_{\mathrm{B}} \widetilde{\complement} \tilde{m}$ Int $\left(\mathrm{F}_{\mathrm{B}}\right)$ whenever $\mathrm{H}_{\mathrm{B}} \widetilde{\complement} \mathrm{F}_{\mathrm{B}}$ and $\mathrm{H}_{\mathrm{B}}$ is sg$\tilde{m}$-closed. Let $\mathrm{F}_{\mathrm{B}}{ }^{\mathrm{C}} \widetilde{\subseteq} \mathrm{U}_{\mathrm{B}}$ and $\mathrm{U}_{\mathrm{B}}$ is sg $\tilde{m}$-open. Then $\mathrm{U}_{\mathrm{B}}{ }^{\mathrm{C}} \widetilde{\subseteq} \mathrm{F}_{\mathrm{B}}$ and $\mathrm{U}_{\mathrm{B}}{ }^{\mathrm{C}}$ is sg $\tilde{m}$-closed. By our

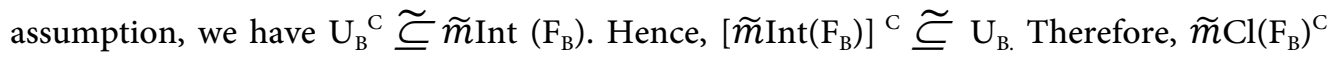
$\widetilde{\complement} \mathrm{U}_{\mathrm{B}}$ Consequently, $\mathrm{F}_{\mathrm{B}}{ }^{\mathrm{C}}$ is sg ${ }^{\star} \widetilde{m}$ closed. Hence, $\mathrm{F}_{\mathrm{B}}$ is sg ${ }^{\star} \widetilde{m}$-open.

Theorem 3.16: If $F_{B}$ and $G_{B}$ are $\operatorname{sg}^{\star} \widetilde{m}$-open sets then $F_{B} \cap G_{B}$ is also sg ${ }^{\star} \widetilde{m}$ open set.

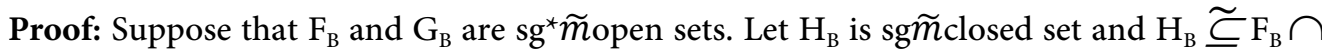
$\mathrm{G}_{\mathrm{B}}$. Since $\mathrm{H}_{\mathrm{B}} \widetilde{\subseteq} \mathrm{F}_{\mathrm{B}} \cap \mathrm{G}_{\mathrm{B}}$, we have $\mathrm{H}_{\mathrm{B}} \widetilde{\subseteq} \mathrm{F}_{\mathrm{B}}$ and $\mathrm{H}_{\mathrm{B}} \widetilde{\subseteq} \mathrm{G}_{\mathrm{B}}$. Since $\mathrm{F}_{\mathrm{B}}$ and $\mathrm{G}_{\mathrm{B}}$ are sg* $\widetilde{m}$ open sets, we have $\mathrm{H}_{\mathrm{B}} \widetilde{\subseteq} \widetilde{m}$-Int $\left(\mathrm{F}_{\mathrm{B}}\right)$ and $\mathrm{H}_{\mathrm{B}} \widetilde{\complement} \widetilde{m}$ Int $\left(\mathrm{G}_{\mathrm{B}}\right)$. Therefore $\mathrm{H}_{\mathrm{B}} \widetilde{\complement} \widetilde{m}$ Int

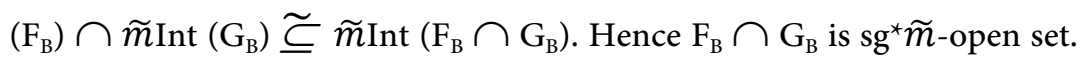

Remark 3.17: The union of two $\operatorname{sg}^{\star} \widetilde{m}$-open sets need not be $\operatorname{sg}^{\star} \widetilde{m}$-open set as can seen from the following example. 
Example 3.18: Let us consider the soft subsets of $\mathrm{F}_{\mathrm{A}}$ that are given in Example 2.3. Let $\left(\mathrm{F}_{\mathrm{A}}, \widetilde{m}\right)$ be a soft minimal space where $\mathrm{U}=\left\{\mathrm{u}_{1}, \mathrm{u}_{2}\right\}, \mathrm{E}=\left\{\mathrm{x}_{1}, \mathrm{x}_{2}, \mathrm{x}_{3}\right\}, \mathrm{A}=\left\{\mathrm{x}_{1}, \mathrm{x}_{2}\right\} \widetilde{\subseteq} \mathrm{E}$ and $\mathrm{F}_{\mathrm{A}}=\left\{\left(\mathrm{x}_{1}\left\{\mathrm{u}_{1}, \mathrm{u}_{2}\right\}\right),\left(\mathrm{x}_{2}\left\{\mathrm{u}_{1}, \mathrm{u}_{2}\right\}\right)\right\}$. Then $\tilde{m}=\left\{\mathrm{F} \Phi, \mathrm{F}_{\mathrm{A} 2}, \mathrm{~F}_{\mathrm{A} 3}, \mathrm{~F}_{\mathrm{A} 8}, \mathrm{~F}_{\mathrm{A} 12}, \mathrm{~F}_{\mathrm{A}}\right\}$. Then sg $\tilde{m}$-closed sets are $\mathrm{F} \Phi, \mathrm{F}_{\mathrm{A} 1}, \mathrm{~F}_{\mathrm{A} 4}, \mathrm{~F}_{\mathrm{A} 6}, \mathrm{~F}_{\mathrm{A} 7}, \mathrm{~F}_{\mathrm{A} 7}, \mathrm{~F}_{\mathrm{A} 9}, \mathrm{~F}_{\mathrm{A} 10}, \mathrm{~F}_{\mathrm{A} 13}, \mathrm{~F}_{\mathrm{A} 14}, \mathrm{~F}_{\mathrm{A}}$ and $\operatorname{sg}^{\star} \tilde{m}$-closed sets are $\mathrm{F} \Phi, \mathrm{F}_{\mathrm{A} 1}, \mathrm{~F}_{\mathrm{A} 6}, \mathrm{~F}_{\mathrm{A} 7}, \mathrm{~F}_{\mathrm{A} 9}, \mathrm{~F}_{\mathrm{A} 10}, \mathrm{~F}_{\mathrm{A} 13}, \mathrm{~F}_{\mathrm{A}}$. sg $^{\star} \widetilde{m}$-open sets are $\mathrm{F}_{\Phi}, \mathrm{F}_{\mathrm{A} 12}, \mathrm{~F}_{\mathrm{A} 3}, \mathrm{~F}_{\mathrm{A} 11}, \mathrm{~F}_{\mathrm{A} 2}, \mathrm{~F}_{\mathrm{A} 8}, \mathrm{~F}_{\mathrm{A} 5}, \mathrm{~F}_{\mathrm{A}}$. Here, $\mathrm{F}_{\mathrm{A} 2}$ and $\mathrm{F}_{\mathrm{A} 8}$ are sg* $\widetilde{m}$-open sets but $\mathrm{F}_{\mathrm{A} 2} \cup \mathrm{F}_{\mathrm{A} 8}=\mathrm{F}_{\mathrm{A} 14}$ is not $\mathrm{sg}^{\star} \widetilde{m}$-open.

Theorem 3.19: Let $F_{B}$ and $G_{B}$ be soft subsets of a soft minimal space $\left(F_{A}, \widetilde{m}\right)$ such that $\widetilde{m}$ Int $\left(\mathrm{F}_{\mathrm{B}}\right) \widetilde{\complement} \mathrm{G}_{\mathrm{B}} \widetilde{\subseteq} \mathrm{F}_{\mathrm{B}}$. If $\mathrm{F}_{\mathrm{B}}$ is sg* $\widetilde{m}$-open, then $\mathrm{G}_{\mathrm{B}}$ is sg ${ }^{\star} \widetilde{m}$ open in $\left(\mathrm{F}_{\mathrm{A}}, \widetilde{m}\right)$.

Proof: Suppose that $\mathrm{F}_{\mathrm{B}}$ and $\mathrm{G}_{\mathrm{B}}$ be soft subsets of $\left(\mathrm{F}_{\mathrm{A}}, \widetilde{m}\right)$. Let $\mathrm{F}_{\mathrm{B}}$ is sg ${ }^{\star} \widetilde{m}$-open set in $\left(\mathrm{F}_{\mathrm{A}}, \widetilde{m}\right)$ such that $\widetilde{m}$ Int $\left(\mathrm{F}_{\mathrm{B}}\right) \widetilde{\complement} \mathrm{G}_{\mathrm{B}} \widetilde{\complement} \mathrm{F}_{\mathrm{B}}$. Let $\mathrm{H}_{\mathrm{B}}$ be a sg $\widetilde{m}$-closed such that $\mathrm{H}_{\mathrm{B}} \widetilde{\complement} \mathrm{G}_{\mathrm{B}}$. Since $\mathrm{H}_{\mathrm{B}} \widetilde{\subseteq} \mathrm{G}_{\mathrm{B}}$, and $\mathrm{G}_{\mathrm{B}} \widetilde{\subseteq} \mathrm{F}_{\mathrm{B}}$, we have $\mathrm{H}_{\mathrm{B}} \widetilde{\subseteq} \mathrm{F}_{\mathrm{B}}$. Since $\mathrm{F}_{\mathrm{B}}$ is sg ${ }^{\star} \widetilde{m}$-open set, we have $\mathrm{H}_{\mathrm{B}} \widetilde{\complement} \widetilde{m}$ Int

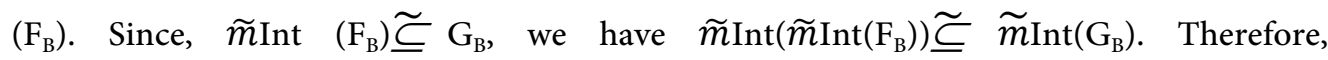

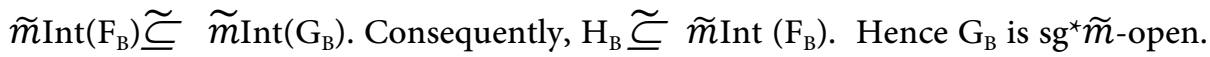

Theorem 3.20: If a soft subset $\mathrm{F}_{\mathrm{B}}$ of a soft minimal space $\left(\mathrm{F}_{\mathrm{A}}, \widetilde{m}\right)$ is sg ${ }^{\star} \widetilde{m}$-closed, then $\tilde{m} \mathrm{Cl}\left(\mathrm{F}_{\mathrm{B}}\right)-\mathrm{F}_{\mathrm{B}}$ is $\mathrm{sg}^{\star} \tilde{m}$-open.

Proof: Suppose that $\mathrm{F}_{\mathrm{B}}$ is sg* $\widetilde{m}$-closed in $\left(\mathrm{F}_{\mathrm{A}}, \widetilde{m}\right)$. Let $\mathrm{H}_{\mathrm{B}} \widetilde{\widetilde{c}} \widetilde{m} \mathrm{Cl}\left(\mathrm{F}_{\mathrm{B}}\right)-\mathrm{F}_{\mathrm{B}}$ and $\mathrm{H}_{\mathrm{B}}$ is $\operatorname{sg} \tilde{m}$ closed. Since $\mathrm{F}_{\mathrm{B}}$ is $\mathrm{sg}^{\star} \tilde{m}$-closed, we have $\tilde{m} \mathrm{Cl}\left(\mathrm{F}_{\mathrm{B}}\right)-\mathrm{F}_{\mathrm{B}}$ does not contain nonempty sg $\tilde{m}$ closed by Theorem 3.11. Consequently, $\mathrm{H}_{\mathrm{B}}=\mathrm{F} \Phi$. Therefore $\mathrm{F} \Phi \simeq \widetilde{\complement} \tilde{m} \mathrm{Cl}\left(\mathrm{F}_{\mathrm{B}}\right)-\mathrm{F}_{\mathrm{B}}, \mathrm{F} \Phi \simeq$ $\tilde{m} \operatorname{Int}\left(\mathrm{mCl}\left(\mathrm{F}_{\mathrm{B}}\right)-\mathrm{F}_{\mathrm{B}}\right)$, we obtain $\mathrm{H}_{\mathrm{B}} \widetilde{\complement} \tilde{m} \operatorname{Int}\left\{\tilde{m} \mathrm{Cl}\left(\mathrm{F}_{\mathrm{B}}\right)-\mathrm{F}_{\mathrm{B}}\right)$. Hence $\tilde{m} \mathrm{Cl}\left(\mathrm{F}_{\mathrm{B}}\right)-\mathrm{F}_{\mathrm{B}}$ is sg* $\tilde{m}$ open.

Theorem 3.21: If a soft subset $F_{B}$ is $\operatorname{sg}^{\star} \widetilde{m}$-open in a soft minimal space $\left(F_{A}, \widetilde{m}\right)$, then $G_{B}$ $=\mathrm{F}_{\mathrm{A}}$ whenever $\mathrm{G}_{\mathrm{B}}$ is sg $\widetilde{m}$-open and $\widetilde{m}$ Int $\left(\mathrm{F}_{\mathrm{B}}\right) \cup \mathrm{F}_{\mathrm{B}} \widetilde{\complement} \mathrm{G}_{\mathrm{B}}$.

Proof: Suppose that $\mathrm{F}_{\mathrm{B}}$ is $\operatorname{sg}^{\star} \tilde{m}$ open in a soft minimal space $\left(\mathrm{F}_{\mathrm{A}}, \tilde{m}\right)$ and $\mathrm{G}_{\mathrm{B}}$ is $\operatorname{sg} \tilde{m}$-open and $\tilde{m}$ Int $\left(\mathrm{F}_{\mathrm{B}}\right) \cup \mathrm{F}_{\mathrm{B}} \widetilde{\complement} \mathrm{G}_{\mathrm{B}}$ implies $\mathrm{G}_{\mathrm{B}}{ }^{\mathrm{C}} \widetilde{\complement} \widetilde{m} \mathrm{Cl}\left(\mathrm{F}_{\mathrm{B}}{ }^{\mathrm{C}}\right)-\mathrm{F}_{\mathrm{B}}{ }^{\mathrm{C}}$. Since $\mathrm{F}_{\mathrm{B}}{ }^{\mathrm{C}}$ is sg${ }^{\star} \widetilde{m}$-closed and $\mathrm{G}_{\mathrm{B}}{ }^{\mathrm{C}}$ is sg $\tilde{m}$-closed. Therefore, $\tilde{m} \mathrm{Cl}\left(\mathrm{F}_{\mathrm{B}}{ }^{\mathrm{C}}\right)-\mathrm{F}_{\mathrm{B}}{ }^{\mathrm{C}}$ contains no nonempty sg $\tilde{m}$-closed set (By Theorem 3.11). Consequently, $\mathrm{G}_{B}{ }^{\mathrm{C}}=\mathrm{F} \Phi$ and hence $\mathrm{G}_{\mathrm{B}}=\mathrm{F}_{\mathrm{A}}$.

Remark 3.22: The converse of the above theorem 3.23 is not true in general as can seen from the following example.

Example 3.23: In Example 3.20, if we take $\mathrm{F}_{\mathrm{B}}=\mathrm{F}_{\mathrm{A} 9}$, then $\tilde{m}$ Int $\left(\mathrm{F}_{\mathrm{B}}\right) \cup \mathrm{F}_{\mathrm{B}} \widetilde{\simeq} \mathrm{F}_{\mathrm{A}}, \mathrm{F}_{\mathrm{A}}$ is sg $\tilde{m}$-open, but $\mathrm{F}_{\mathrm{B}}=\mathrm{F}_{\mathrm{A} 9}$ is not sg ${ }^{\star} \widetilde{m}$-open. 


\section{Conclusion:}

In the present work, we have introduced soft $\mathrm{g}^{\star}$-closed and open sets in soft minimal spaces which are defined over an initial universe with a fixed set of parameters. We have explored some basic properties of these concepts. Also, we have established several interesting results and presented its fundamental properties with the help of some examples. In future, these findings may be extended to new types of soft generalized closed and open sets in soft minimal spaces.

\section{Reference:}

[1] Boonpok, Biminimal Structure Spaces, International Mathematical Forum, 15 (5) (2010) $703-707$.

[2] Cammaroto and Noiri, on $\wedge$ - sets and related topological spaces, Acta Math. Hungar, 109 (3) (2005) 261279.

[3] Fukutake, on generalized closed sets in bitopological spaces, Bull. Fukuoka Univ. Ed. Part III, 35 (1986) 1928.

[4] Gowri and Vembu, Soft minimal and soft biminimal spaces, Int. Jr. of Mathematical Science and Appl., Vol. 5, No.2, (2015) 447-455.

[5] Gowri and Vembu, soft g-closed sets in soft minimal spaces, Int. Jr. of Mathematics and Computer Research, Vol. 4, (2016) 1563-1571.

[6] Ittanagi, Soft Bitopological Spaces, International Journal of Computer Applications, Vol. 107, No.7, (2014)

[7] Kelly, Bitopological Spaces, Proc. London Math. Soc., 13 (1963) 71-81.

[8] Levine, Generalized closed sets in topology, Rend. Circ. Mat. Palermo (2), 19 (1970) 89-96.

[9] Molodtsov, Soft Set Theory First Results. Computer and Mathematics with Applications, Vol.37 (1999) 1931 .

[10] Noiri and Popa, A generalized of some forms of g-irresolute functions, European Jr. of Pure and Appl. Math., 2(4) (2009) 473-493.

[11] Noiri, A unified theory for certain modification of generalized form of continuity under minimal condition, Mem. Fac. Sci. Kochi. Univ. ser. A. Math., 22 (2001) 9-18.

[12] Popa and Noiri, On M-continuous functions, Anal. Univ. Dunarea de Jos Galati, Ser. Mat. Fiz. Mec. Teor., Fasc. II, 18, No. 23 (2000) 31-41.

[13] Shabir and Naz, On Soft Topological Spaces, Computers and Mathematics with Applications, 61 (2011) 1786-1799.

[14] Viriyapong et al, Generalized m-Closed Sets in Biminimal Structure Spaces, Int. Jr. of Math. Analysis, Vol.5, No.7, (2011) 333-346. 


\section{Authors' Profile:}

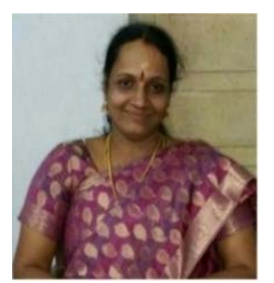

Dr. R. Gowri was born in Kumbakonam, Thanjavur, Tamil Nadu, India in 1978. She received her B.Sc., M.Sc., and M.Phil degree in Mathematics from Bharathidasan University, Tiruchirappalli, India. She worked as a lecturer in Idhaya College for Women, Kumbakonam in the period of 2002-2003. In 2003, she joined as a lecturer in the Srinivasa Ramanujan Research Centre, SASTRA University and later got promoted as a Assistant Professor. She did her research work in SASTRA University and received her Ph.D in 2009. Then she joined as an Assistant Professor in Department of Mathematics, Government College for Women(A), Kumbakonam, Thanjavur, India in 2011. Her areas of Interest are Topology and Graph Theory. She published more than 47 research papers in various International/National Journals. Currently Eight Research Scholars are doing Ph.D under her guidance out of three them have submitted their Ph.D Thesis.

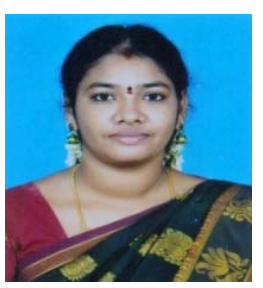

Mrs. S. Vembu was born in Thanjavur, Tamil Nadu, India in 1987. She received her B.sc., M.Sc. and M.Phil. degree in Mathematics from the Bharathidasan University, Trichirappalli in 2007, 2011 and 2012 respectively, B.Ed degree in Mathematics from the Tamilnadu Teacher Education University, Chennai in 2009. She is doing her $\mathrm{Ph} . \mathrm{D}$ under the guidance of Dr. R. Gowri , Assistant Professor, Department of Mathematics, Government College for Women(A), Kumbakonam. Her main area of research interest is Topology. 